\title{
Predictor Design for Altitude Control of a Seaweed Harvester
}

\author{
Marco Gallieri* John Ringwood ** \\ Andrea Giantomassi ${ }^{* * *}$ Gianluca Ippoliti ${ }^{* * *}$ Sauro Longhi ${ }^{* * *}$ \\ * Institute for Systems and Robotics (ISR), Instituto Superior Técnico \\ (IST), Lisbon, Portugal. \\ Email: gallieri.marco@gmail.com \\ ** Department of Electronic Engineering, National University of \\ Ireland, Maynooth, Co. Kildare. \\ Email: john.ringwood@eeng.nuim.ie \\ *** Dipartimento di Ingegneria, Informatica, Gestionale e \\ dell'Automazione, Universitá Politecnica delle Marche, Ancona, Italy. \\ Email: \{a.giantomassi, gianluca.ippoliti,sauro.longhi\}@univpm.it
}

\begin{abstract}
In this paper, the predictor design, for altitude control of a seaweed harvester, is investigated. The harvesting system consists of a vessel and a suspended harvester device, the altitude of which is controlled by a winch. The control approach of Gallieri and Ringwood (2010), including a feedforward action, which requires a single step disturbance prediction, is investigated further, focusing on the disturbance prediction, for noisy sensors. The prediction is performed using AR and ARMA models, identified online, by using the Recursive Least Squared with Forgetting Factor (RLSFF) algorithm and the Kalman Filter (KF). The dependance between the error spectrum and the quality of the control is shown, and the prediction performances are evaluated, using an FFT-based criterion, oriented to the feedforward application. The control performances are then evaluated, and the results are compared to Gallieri and Ringwood (2010).
\end{abstract}

Keywords: Marine systems, Feedforward compensation, Linear prediction, Kalman filters, Recursive least squares, Spectral analysis

\section{INTRODUCTION}

The seaweed harvester system under consideration is shown in Figure 1. The challenge of maintaining a suspended device at a specific distance from an undulating sea bed is new, though the adopted solution draws upon considerable prior art in the area of marine systems and hydrodynamics, Gallieri and Ringwood (2010); Gallieri $(2009 a, b)$ and reference therein. In particular, the body of knowledge available on offshore cranes is particularly relevant: Falat et al. (2005); Messineo et al. (2008); Sagatun et al. (2003). In the previous work, Gallieri and Ringwood (2010), a detailed hydrodynamic model has been formulated for the vessel/harvester system, and a control strategy has been proposed, including a vessel motion feedforward action, using a Motion Reference Unit (MRU), and an altitude feedback loop, using a sonar device for altitude measurement. In particular, Gallieri and Ringwood (2010), shown the feasibility of the harvester altitude control within $\pm 10 \mathrm{~cm}$. This is necessary in order to maintain an optimum cutting height while avoiding any environmental damage to the seabed. The ship is subject to ocean wave disturbances, and moves at an average constant speed, with a periodical turning manoeuvre, in relatively shallow water.

The feed-forward control strategy, proposed in Gallieri and Ringwood (2010), which compensates the heave variations

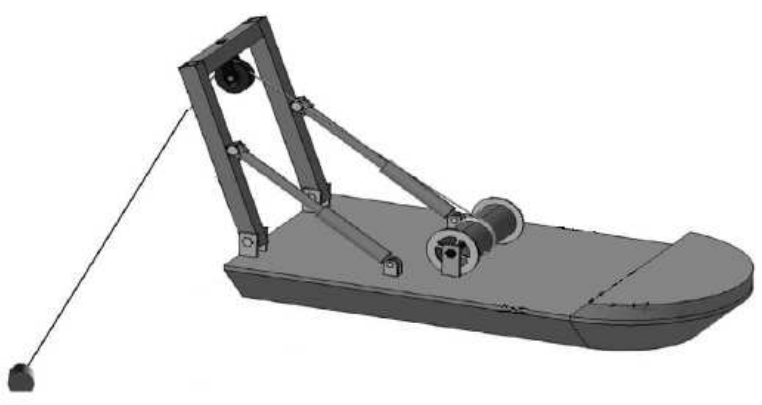

Fig. 1. The seaweed harvester, Gallieri and Ringwood (2010)

in the harvester due to the wave motions experienced by the vessel, requires a one-step prediction. The signal to predict is the nonlinear motion of the harvester, induced by the vessel in ocean waves. The seastate is represented as a stochastic process finite realization, defined by a JONSWAP spectrum, Fossen (2002), with a directional spreading factor, Perez (2005). In this paper, linear AR and ARMA models are proposed as predictor candidates, while a Recursive Least Squares with Forgetting Factor (RLSFF) and a Kalman Filter (KF) are used for the model parametrization. The AR, ARMA are parametrized online, unlike in Gallieri and Ringwood (2010), where the identification has been performed on a fixed sized samples window. 
The feedforward control performances depends on the prediction error dynamics. In particular, the high frequency components are filtered by the control law. The proposed candidate model classes and estimation techniques are compared, first by computing the prediction Root of Mean Squared Error (RMSE), and then computing the error spectrum Low Frequency components RMSE (LFRMSE). The control performances are evaluated using again the RMSE, and the IAE and ISE integral indexes. The prediction and control results are discussed, and compared to Gallieri and Ringwood (2010), featuring a Periodical Least Squared AR (PLSAR) predictor. A simulator, obtained by modifying the Simulink MSS Toolbox, MSS (2010), is used to evaluate the estimation and control performances.

The paper addresses the problem as follows: Section 2 summarizes the dynamics of the vessel and the winch system, along with any simplifying modelling assumptions. Section 3 details the feedforward/feedback control design, while Section 4 issues the predictor design and the used identification techniques. Section 5 summarizes the prediction and control results. Finally, conclusions are drawn in Section 6.

\section{VESSEL AND WINCH DYNAMICS}

The considered ship and harvester model, has 9 degree of freedom (DOF), and its reference frames are defined in Gallieri and Ringwood (2010). The coordinates, used to describe the system dynamics, are defined by the Society of Naval Architects and Marine Engineers (SNAME), and shown in Table 1.

\begin{tabular}{|c|c|}
\hline Variable name & Description \\
\hline$n_{i}, e_{i}, d_{i}$ & North, east, down positions, $n$-frame \\
\hline$\phi_{i}, \theta_{i}, \psi_{i}$ & Roll, pitch, yaw (Euler) angles, $n \rightarrow b_{i}$ \\
\hline$u_{i}, v_{i}, w_{i}$ & Surge, sway, heave velocities, $b_{i}$-frame \\
\hline$p_{i}, q_{i}, r_{i}$ & Roll, pitch, yaw rate, $b_{i}$-frame \\
\hline$z_{m}^{b_{2}}, v_{m}^{b_{2}}$ & Linear motor position and velocity, $b_{2}$-frame \\
\hline$v_{d}^{b_{2}}$ & Motor velocity input, $b_{2}$-frame \\
\hline$q, s$ & Generalized displacements and velocities \\
\hline$P$ & Colocity transformation matrix, $b \rightarrow$ NEEC \\
\hline$v_{c_{i}}^{b_{i}}$ & CoG angular velocity vector, $n \rightarrow b_{i}$ \\
\hline$\omega_{n}^{b_{i}}$ & Velocity vector, $b$-frame \\
\hline$\nu$ & Position vector, $n$-frame \\
\hline$\eta$ & State, output vectors \\
\hline$\underline{x}, \underline{\mathrm{y}}$ & Fluid memory state, NEEC reference \\
\hline$\mu$ & Vessel forward speed \\
\hline$U$ & Control input vector \\
\hline$U_{i n}$ & State, measurement noise vectors \\
\hline$W_{x}, W_{y}$ & Harvester heave disturbance estimation, $n$-frame \\
\hline$e(k), \hat{e}(k)$ & Altitude control and estimation errors, $n$-frame \\
\hline$T_{s}$ & Harvester heave measurement noise, $n$-frame \\
\hline$d w$ &
\end{tabular}

The 9 DOF dynamical model, detailed in Gallieri and Ringwood (2010), includes the effects of a forward vessel speed, the wave loads, the ship propellers and motion control forces, and the winch motor dynamics. This formulation arises from the unified ship model, Perez (2005), and includes the seakeeping fluid memory effects, where a state space approximation of the Cummins' equation, Cummins
(1962), is computed for both the vessel and the harvester device, using the approach in Kristiansen and Egeland (2003). From a control prospective, using the modelling approach of Perez (2005), the control design becomes more challenging, because the wave induced disturbance is considered to be acting on the system input, rather than the output. To model the linear wave-excitation forces, the force response amplitude operator (Force-RAO) Perez (2005), is used, consisting of a set of linear transfer functions. The hydrodynamic parameters and the ForceRAO are computed in WAMIT, Wamit (2008), for both the vessel and the harvester device, and processed using the Matlab/Simulink MSS toolbox, MSS (2010).

The modelling approach, presented in Gallieri and Ringwood (2010), is a more general extension of the formulation of Gallieri and Ringwood (2009), where the coupled dynamics of the vessel and the harvester are formulated by using the Newton-Euler equations with Eliminated Constraints (NEEC), as in O'Cathain et al. (2008). The harvester, the specific design of which is currently confidential, is assumed to be a vertical circular cylinder.

\subsection{Modelling assumptions}

The system dynamics are formulated under the assumption that the $b_{2}$-frame dynamics, between the real motor speed $v_{m}^{b_{2}} \equiv \dot{z}_{m}^{b_{2}}$ and the desired motor speed $v_{d}^{b_{2}}$, is given by the following first order LTI system, after Messineo et al. (2008); Sagatun et al. (2003):

$$
\dot{v}_{m}^{b_{2}}=[-\lambda] v_{m}^{b_{2}}+[\lambda] v_{d}^{b_{2}}
$$

where $\frac{1}{\lambda}$ is the time constant of the closed loop speed servo system. The servomotor has an incremental encoder, which measures the drum rotational speed, and the measurement noise is assumed to be white. The vessel is equipped with a motion reference unit (MRU 5) Seatex (2006), an accelerometer-based position, velocity and acceleration estimation device, which uses an ad-hoc Kalman filter, Jazwinski (1970), and provides the measurement, of the vessel roll, pitch and yaw angles, in the vehicle frame, and the relative heave, surge and sway positions. The MRU measurement noise is also modelled as a zero mean white noise, where the variance is given by the user manual, Seatex (2006). The harvester device is equipped with a sonar device, which senses the instantaneous seabed profile. To simulate the real sonar measurement, it's assumed that the sonar has a cone-shaped beam dispersion surface, with a dispersion angle $\alpha_{s}$, and an additional (white) measurement noise, Gallieri (2009b). In this study, the stiffness of the cable connecting vessel and harvester is assumed to be infinitely stiff.

\subsection{State space representation}

Expanding the formulation of Perez (2005), a 2-body unconstrained 12 DOF motion model can be obtained, as in Gallieri and Ringwood (2010); Gallieri (2009b). Then, using the NEEC approach, the model presented here will have $9 \mathrm{DOF}$. To apply the NEEC, the following $9 \times 1$ independent velocity vector (generalized coordinates) is choosen:

$$
s=\left[\begin{array}{lllllllll}
u_{1} & v_{1} & w_{1} & p_{1} & q_{1} & r_{1} & p_{2} & q_{2} & \dot{z}_{m}
\end{array}\right]^{T},
$$


defined in Table 1, where the subscripts 1 and 2 denote, respectively, the vessel and the harvester. The partial velocity matrix, $P \in \mathbb{R}^{9 \times 12}$, given by:

$$
P=\left[\left(\frac{\partial v_{c_{1}}^{b_{1}}}{\partial s}\right)^{T}\left(\frac{\partial \omega_{n b_{1}}^{b_{1}}}{\partial s}\right)^{T}\left(\frac{\partial v_{c_{2}}^{b_{2}}}{\partial s}\right)^{T}\left(\frac{\partial \omega_{n b_{2}}^{b_{2}}}{\partial s}\right)^{T}\right]
$$

is defined. The generalized velocities, $\nu$, and accelerations $\dot{\nu}$, are given by:

$$
\nu=P^{T} s \dot{\nu}=P^{T} \dot{s}+\dot{P}^{T} s
$$

Defining the vector $q$, such that $\dot{q}=s$, where $s$ is given by eq.(2), the following state, input and output vectors can be defined respectively as:

$$
\underline{\mathrm{x}}=\left[\begin{array}{c}
q \\
s \\
\mu \\
\eta
\end{array}\right], \quad U_{i n}=\left[\begin{array}{c}
\left(\tilde{\tau}_{c}+\tilde{\tau}_{p}\right) \\
v_{d}
\end{array}\right], \quad \mathbf{y}=\left[\begin{array}{c}
\tilde{s} \\
\tilde{\eta}
\end{array}\right],
$$

where:

$$
\tilde{s}=\left[\begin{array}{lllllll}
u_{1} & v_{1} & w_{1} & p_{1} & q_{1} & r_{1} & w_{2}
\end{array}\right]^{T},
$$

and $\underline{\mathrm{x}} \in \mathbb{R}^{84 \times 1}, U_{\text {in }} \in \mathbb{R}^{10 \times 1}, \underline{\mathrm{y}} \in \mathbb{R}^{14 \times 1}$. The vectors $\tilde{\tau}_{c}$ and $\tilde{\tau}_{p}$, provide the generalized propeller and ship motion control forces in the $b$-frames, as given in Perez (2005) and Fossen (2002), and $v_{d}$, the desired winch motor speed, is the altitude control (manipulated) signal.

The seaweed harvester dynamics are given by the following non-linear stochastic state space system, Gallieri and Ringwood (2010):

$$
\begin{gathered}
\underline{\dot{\mathrm{x}}}=A(\underline{\mathrm{x}}) \underline{\mathrm{x}}+B(\underline{\mathrm{x}}) U_{i n}+W_{x} \\
\underline{\mathrm{y}}=C(\underline{\mathrm{x}}) \underline{\mathrm{x}}+W_{y},
\end{gathered}
$$

where the matrices, $B(\underline{\mathrm{x}}) \in \mathbb{R}^{84 \times 10}, A(\underline{\mathrm{x}}) \in \mathbb{R}^{84 \times 84}$, are given in Gallieri and Ringwood (2010).

The state disturbance vector, $W_{x} \in \mathbb{R}^{84 \times 1}$, appearing in (7) and due to the first and second order wave forces Journee and Massie (2001), is given by:

$$
W_{x}=\left[\begin{array}{llll}
0 & \left(\widetilde{\tau}_{w}^{g}\right)^{T} & 0 & 0
\end{array}\right]^{T}
$$

where $\widetilde{\tau}_{w}^{g}$ contains the first 8 elements of the first and second order wave induced force vector, $\tau_{w}^{g}$ Perez (2005), which is computed using the RAO approach as in Perez (2005) and Journee and Massie (2001). The output disturbance vector, $W_{y} \in \mathbb{R}^{14 \times 1}$, in $(7)$, is a zero mean white noises vector, with unknown variance. The output matrix, $C(\underline{\mathrm{x}}) \in \mathbb{R}^{14 \times 84}$, in $(7)$, is given by:

$$
C(\underline{\mathrm{x}})=\left[\begin{array}{cccc}
0 & C_{s} & 0 & 0 \\
0 & 0 & 0 & C_{\eta}(\underline{\mathrm{x}})
\end{array}\right]
$$

where $C_{s}$ and $C_{\eta}(\underline{\mathrm{x}})$, are given in Gallieri (2009a,b).

\section{CONTROL DESIGN}

For the altitude control of the harvesting device, three control strategies have been implemented and compared, in Gallieri and Ringwood (2010), where the best performances have been achieved by using a combined control strategy. The proposed combined strategy, shown in Figure
2, which includes a feed-forward controller to counteract the vessel-induced altitude motion of the cutter, and an altitude feedback controller, to follow the seabed profile. The feed-forward scheme, shown in Figure 2, uses the Seatex MRU and the seaweed harvester kinematics to compute the nonlinear motion of the harvester, induced by the vessel, which is the input for the feed forward (FF) controller. Assuming that the motor dynamics are linear, the FF control law is a LTI discrete time transfer function.

The wave induced disturbance, $d(k)$, acting on the harvester altitude, is a function of the system state $\underline{x}$. To compute the current value of $d(k)$, an estimator is needed, as shown in Section 3.1. The estimation error affects the quality of the control, as shown in Section 4.3. The feedback scheme, shown in Figure 2, uses the sonar device to sense the distance between the harvester and the seabed. A Nomoto PID vessel heading controller, Perez (2005), included in the MSS toolbox, is used for course keeping.

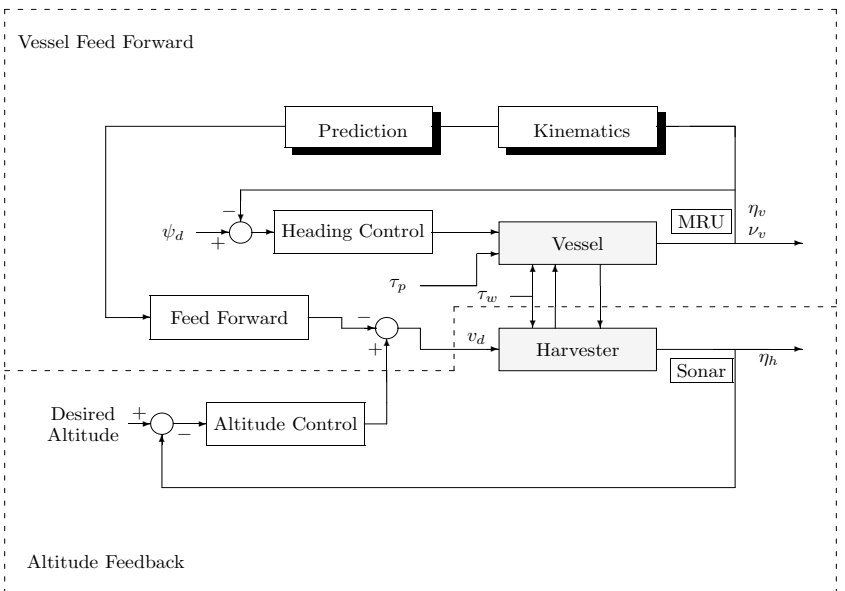

Fig. 2. The altitude control scheme

\subsection{The feed-forward vessel motion compensator}

The harvester motion satisfies the following kinematic relation, Gallieri (2009b):

$$
\begin{aligned}
\dot{z}_{2} & =R_{2}^{n} w_{2} \\
& =\operatorname{Row}_{3}\left\{R_{1}^{n}\right\}\left[\begin{array}{c}
u_{1} \\
v_{1} \\
w_{1}
\end{array}\right]+\operatorname{Row}_{3}\left\{R_{1}^{n} S^{T}\left(r_{o_{2}}^{b_{1}}\right)\right\}\left[\begin{array}{c}
p_{1} \\
q_{1} \\
r_{1}
\end{array}\right] \\
& +\operatorname{Row}_{3}\left\{R_{2}^{n}\right\}\left[\begin{array}{c}
l_{c_{2}} q_{2} \\
-l_{c_{2}} p_{2} \\
v_{m}
\end{array}\right]
\end{aligned}
$$

Assuming that the position, velocity and acceleration of the vessel are measurable, taking the small angle approximation for the harvester motion, and substituting the results of eq.(13), we obtain the following model:

$$
\begin{gathered}
\dot{v}_{m}(t)=-\lambda v_{m}(t)+\lambda v_{d} \\
\dot{z}_{2}(t)=v_{m}(t)+d(t)+d_{w}(t),
\end{gathered}
$$

where

$$
\begin{aligned}
d(t)= & -s \theta_{1} u_{1}+c \theta_{1} s \phi_{1} v_{1}+c \theta_{1} c \phi_{1} w_{1}-\bar{z}_{O_{2}} c \theta_{1} s \phi_{1} p_{1} \\
& -\left[\bar{z}_{O_{2}} s \theta_{1}+\bar{x}_{o_{2}} c \theta_{1} c \phi_{1}\right] q_{1}+\left[\bar{x}_{o_{2}} c \theta_{1} s \phi_{1}\right] r_{1},
\end{aligned}
$$

is the vessel induced heave velocity disturbance, which is a function of the vessel attitude and speed, while $d_{w}(t)$ is a Gaussian zero mean white noise disturbance, including 
the model uncertainties and the measurement noise. From eq.(11), we have:

$$
\begin{gathered}
v_{m}(s)=\frac{\lambda}{s+\lambda} v_{d} \\
z_{2}(s)=\frac{1}{s}\left(v_{m}(s)+d(s)+d_{w}(s)\right) .
\end{gathered}
$$

Choosing the following feed-forward control law:

$$
v_{d}(s)=-\gamma \frac{s+\lambda}{\lambda(s+\gamma)} d(s)
$$

the system dynamic becomes:

$$
z_{2}(s)=\frac{1}{s+\gamma} d(s)+\frac{1}{s} d_{w}(s)
$$

\subsection{The gain scheduled feedback loop}

Combining the predictive heave feed-forward, shown in Section 3.1, with a gain scheduling $\mathrm{P}$ controller, we obtain the following control law:

$$
v_{d}(k)=\tilde{v}_{d}(k)+v_{d}^{\star}(k),
$$

where $\tilde{v}_{d}(k)$ is given by the feed forward controller, a discrete-time Tustin's equivalent of eq.(15), as shown in Gallieri and Ringwood (2010), and:

$$
v_{d}^{\star}(k)=K_{p} e(k),
$$

where $K_{p}$ is adapted with the rule:

$$
K_{p}=\left\{\begin{array}{cl}
K_{p}^{M A X}, & |e(k)| \geq e^{\star} \\
\left(K_{p}^{M A X}-K_{p}^{M I N}\right) \frac{|e(k)|}{e^{\star}}+K_{p}^{M I N}, & |e(k)|<e^{\star}
\end{array},\right.
$$

where $e(k)$ is the altitude error, and with $e^{\star}=0.1 \mathrm{~m}$, and $K_{p}^{M A X}=3, K_{p}^{M I N}=1.5$.

\section{PREDICTOR DESIGN}

\subsection{Candidate model classes}

The estimation will be performed by a dynamical model, identified online. The following candidate model classes have been chosen:

- AutoRegressive (AR):

$$
\hat{d}(k)=-a_{1} d(k-1)-\ldots-a_{n} d(k-n),
$$

- AutoRegressiveMovingAverage (ARMA):

$$
\begin{aligned}
\hat{d}(k)= & -a_{1} d(k-1)-\ldots-a_{n} d(k-n)+ \\
& c_{1} \hat{e}(k-1)+\ldots+c_{n} \hat{e}(k-n) .
\end{aligned}
$$

\subsection{Identification approaches}

Two linear regressors are compared:

- Recursive Least Squares with Forgetting Factor (RLSFF), Ljung (1999): for the AR identification, a forgetting factor, $\sigma$, is used, to deal with time varying parameters. The significative samples for the estimation algorithm are contained in a window of length:

$$
N_{s}=\frac{1}{(1-\sigma)} \text {. }
$$

For the AR model $\sigma=0.999$ is used, having $N_{s}=$ 1000, while for the ARMA model, the Recursive
Extended Least Squares (RELSFF), Ljung (1999), are used, with $\sigma=0.99$, and $N_{s}=100$. The polynomial instability has been avoided using the roots reflection into the unit circle, Ljung (1999).

- Kalman Filter (KF): Kalman filter is used for parameter estimation, as in Jazwinski (1970); Anderson and Moore (1979). Tests are made to tune the covariance matrices of the process and measurements noise. As shown in Fioretti and Jetto (1994), it is possible to put the process noise covariance, in the form:

$$
Q=q I
$$

where $I$ is the identity matrix. The measurement noise variance $r$ is also a scalar. The optimal values for $q$ and $r$ are determined by their ratio, $q / r$, as in Fioretti and Jetto (1994), instead of the absolute value. This value is determined by trial and error, considering the signal dynamics and the measurement noise. Considering that, from experimental results, the model parameters are slowly varying, the measure uncertainties are dominant, determining a small $q / r$ ratio. Best results are achieved by setting $q / r \approx 10^{-6}$.

The AR and ARMA model order can be chosen with an appropriate data analysis. The spectral norm of the observation matrix is used to provide a lower bound, while the cross validation is performed, to determine the correct complexity, Ljung (1999). To optimize the accuracy/memory ratio, the MDL index is preferred, as in Ljung (1999). A sample of the tests made to optimize the model order is shown in Figure 3, for the AR model. The chosen predictors are: AR 9, $\operatorname{ARMA}(9,9)$.

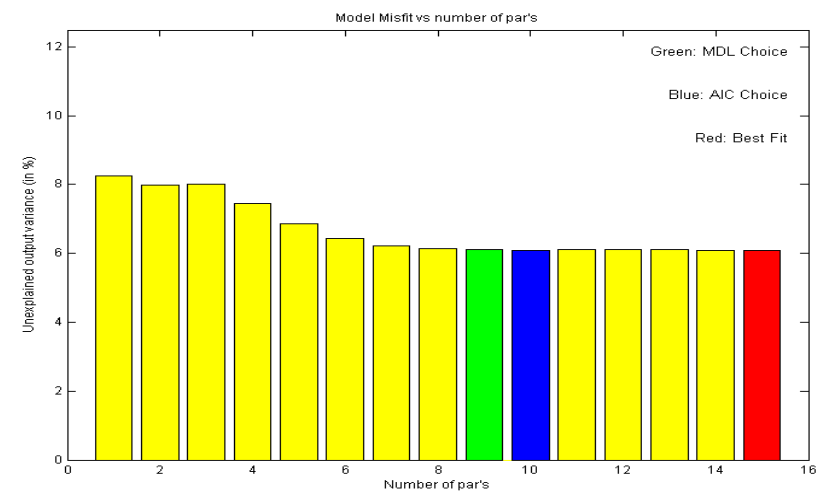

Fig. 3. Results of MDL, AIC and Best Fit tests for the AR model order choice.

\subsection{Performance indexes}

In Gallieri and Ringwood (2010), the prediction performances have been evaluated using the RMSE index, which can provide only partial information about the estimation influence on the control performances. The prediction residual error dynamics has, in fact, a strong influence on the quality of the feedforward control, and therefore of the altitude control. This can be shown, by expressing the prediction as $\hat{d}(k)=d(k)-\hat{e}(k)$, and substituting eq. $(15,13)$ into eq.(14), obtaining the following feedforward controlled harvester heave dynamic: 


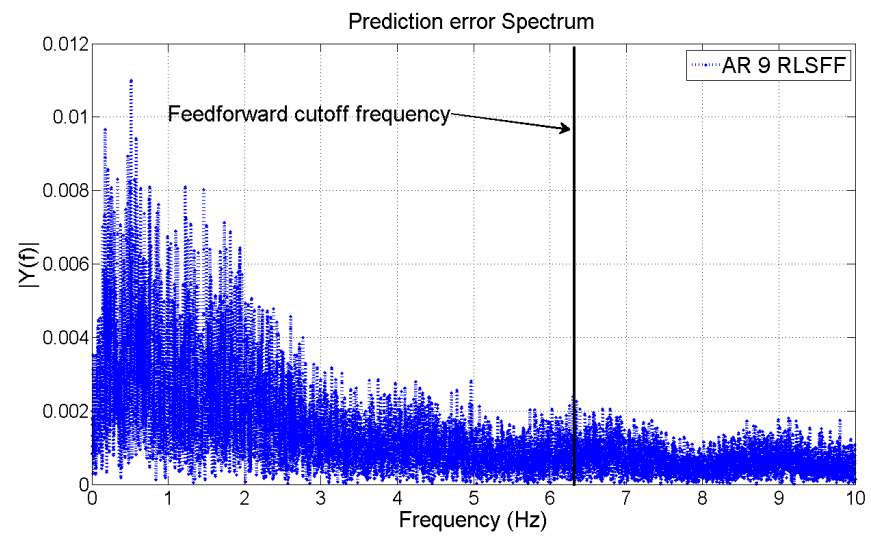

Fig. 4. Estimation error spectrum (RLSFF AR 9), only the low frequency components affect the control

$$
\begin{gathered}
z_{2}(s)=\frac{1}{s}\left(G_{1}(s) d(s)+G_{2}(s) \hat{e}(s)+d_{w}(s)\right), \\
G_{1}(s)=\frac{s}{s+\gamma}, G_{2}(s)=\frac{\gamma}{s+\gamma},
\end{gathered}
$$

where the transfer function $G_{2}(s)$ between the estimation error and the heave velocity, is a low pass filter and its cutoff frequency, $\gamma$ is a project parameter, in this case $\gamma=40[\mathrm{rad} / \mathrm{sec}] \approx 6.3[\mathrm{~Hz}]$. From the spectrum of the LRSFF AR prediction error, shown in Fig.4, it's possible to see that the AR prediction has some residual low frequency dynamics.

From the above considerations, we will evaluate the prediction performance using both the RMSE, and the following index:

$$
\operatorname{LFRMSE}=\operatorname{RMS}\left(X^{L F}(j \omega)\right)
$$

where $X(j \omega)$ is the amplitude spectrum of the prediction error, computed using the Fast Fourier Transform (FFT), while the subscript LF indicates that only the components having $\omega<\omega_{c}$ are considered, where $\omega_{c}$ is the dominant pole frequency of the LTI filter, in this case $G_{2}(s)$, from eq. (24), excluding $s=0$. The LFRMSE provides a comparison index, between the prediction strategies, as a raw measure of how much the prediction error will be filtered out, preventing the control error increase. The use of the LFRMSE can be extended to similar applications, whereas an estimation is followed by a low-pass LTI dynamics.

To evaluate the control performances we use the RMSE, and the following indexes, as in Hsiao-Ping and YungCheng (1982): the Integral of Absolute Error (IAE), which penalizes the control error, and the Integral of Squared Error (ISE), which penalizes the error peakedness.

\section{RESULTS}

The simulated system is subject to variations in free surface elevation and seabed profile, as shown in Fig.5. The surface variations come from a JONSWAP, Hasselmann and Olbers (1973), model with a significant wave height of $1.5 \mathrm{~m}$, a directional spreading factor of 4, Fossen (2002), and a mean relative wave direction of $\pi$ rads/s. The vessel keeps an average forward speed of $0.5 \mathrm{~m} / \mathrm{s}$. The seabed variations constitute both abrupt and smooth changes, with a variance of $2.15 \mathrm{~m}$ around the mean seabed height. The prediction and control results are

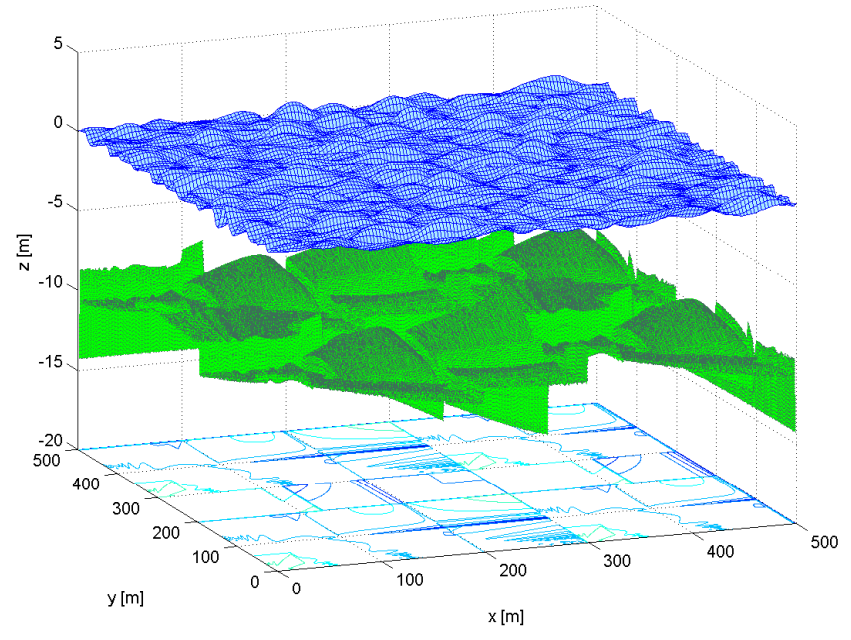

Fig. 5. Variations in seabed profile and free surface elevation

summarized, respectively, in Table 2, 3, for noisy sensors. The results are compared to the PLSAR, presented in Gallieri and Ringwood (2010). Figure 6 shows the RLSFF AR prediction, while Fig.7 shows the convergence of the prediction RMSEs, after $350 \mathrm{sec}$. Considering Table 2, 3

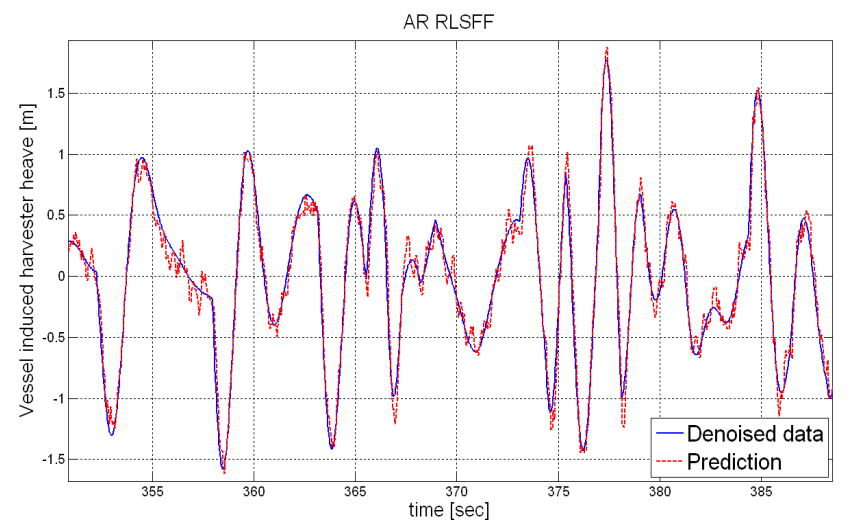

Fig. 6. Disturbance prediction, using the RLSFF AR approach

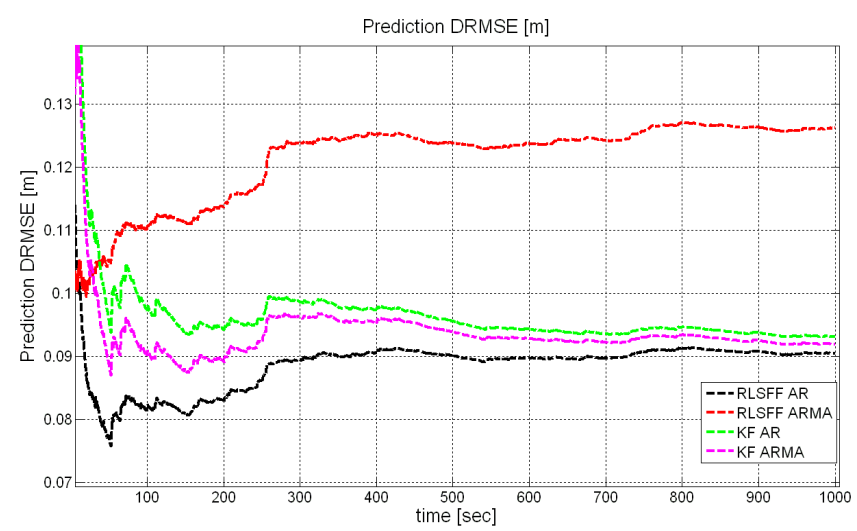

Fig. 7. Prediction RMSE vs simulation time

it's possible to see that, the KF ARMA model provides a prediction RMSE smaller than the KF AR model, versus a bigger control IAE, ISE and RMSE. On the other hand, the LFRMSE index behavior agrees with the control results, for all the considered approaches. The best control 
performances, shown in Figure 8, are achieved using an RLSFF AR 9, with an RMSE of $3.76[\mathrm{~cm}]$ versus the $8[\mathrm{~cm}]$ of the PLSAR. However, as linear regressors, the RLSFF and KF offers very similar results, for continuously varying parameters, and a different parametrization could state the $\mathrm{KF} \mathrm{AR}$ as the best predictor.

\begin{tabular}{|c|c|c|}
\hline Strategy & RMSE & LFRMSE \\
\hline AR (RLSFF) & $0.0901[\mathrm{~m}]$ & $6.729510^{-4}[\mathrm{~m}]$ \\
\hline ARMA (RELSFF) & $0.1238[\mathrm{~m}]$ & $7.251110^{-4}[\mathrm{~m}]$ \\
\hline AR (KF) & $0.0969[\mathrm{~m}]$ & $7.479310^{-4}[\mathrm{~m}]$ \\
\hline ARMA (KF) & $0.0947[\mathrm{~m}]$ & $7.820310^{-4}[\mathrm{~m}]$ \\
\hline PLSAR & $0.1555[\mathrm{~m}]$ & $0.019[\mathrm{~m}]$ \\
\hline
\end{tabular}

Table 2. Prediction performances

\begin{tabular}{|c|c|c|c|}
\hline Strategy & IAE & ISE & RMSE \\
\hline AR (RLSFF) & $8.9108[\mathrm{~m}]$ & $0.7062[\mathrm{~m}]$ & $0.0376[\mathrm{~m}]$ \\
\hline ARMA (RELSFF) & $10.2238[\mathrm{~m}]$ & $0.7827[\mathrm{~m}]$ & $0.0396[\mathrm{~m}]$ \\
\hline AR (KF) & $10.8082[\mathrm{~m}]$ & $0.8371[\mathrm{~m}]$ & $0.0409[\mathrm{~m}]$ \\
\hline ARMA (KF) & $11.0121[\mathrm{~m}]$ & $0.8435[\mathrm{~m}]$ & $0.0411[\mathrm{~m}]$ \\
\hline PLSAR & $17.5438[\mathrm{~m}]$ & $1.7103[\mathrm{~m}]$ & $0.08[\mathrm{~m}]$ \\
\hline
\end{tabular}

Table 3. Control performances

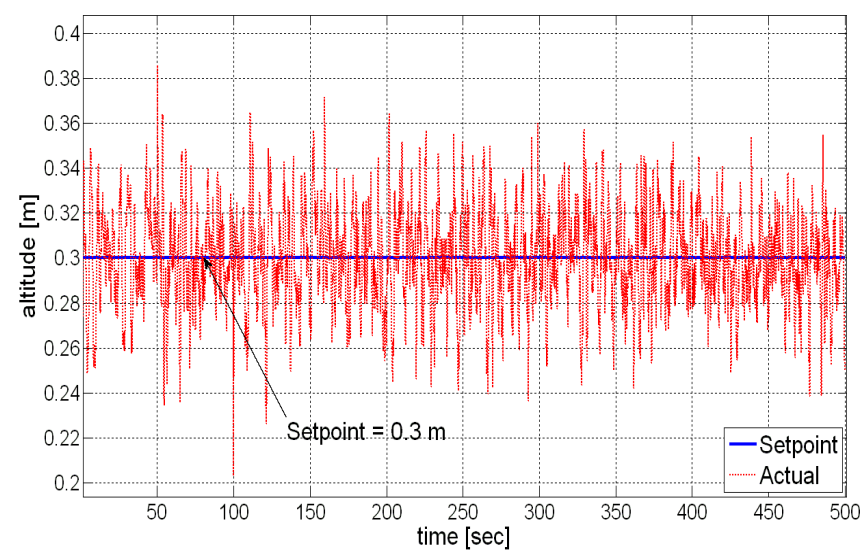

Fig. 8. Altitude control, with an RLSFF AR 9 predictor

\section{CONCLUSION}

In this paper, the predictor design for heave compensation and altitude control of a seaweed harvester, in ocean waves, with non-ideal sensors, has been investigated. The dependance of the the control performances on the estimation error spectrum has been addressed, and the quality of the control is only affected by the low frequency error components. The best control performances are achieved by using an RLSFF AR 9 model, with an IAE of $8.9108[\mathrm{~m}]$, in $500 \mathrm{sec}$, and an RMSE of $3.76[\mathrm{~cm}]$. Practical tests will be addressed, in cooperation with BioAtlantis LTD.

\section{ACKNOWLEDGEMENTS}

The first author acknowledges the financial support of

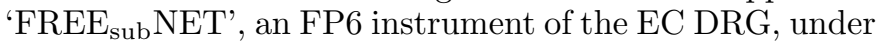
contract number MRTN-2006-036186.

\section{REFERENCES}

Anderson, B. and Moore, J. (1979). Optimal Filtering. Prentice Hall, New York, USA.

Cummins, W. (1962). The impulse responce function and ship motion. Technical Report 1661, David Taylor Model Basin-DTNSRDC.
Falat, P., Brzozowska, L., and Brzozowski, K. (2005). Application of neural network to control the load motion of an offshore crane. In IEEE Workshop on Intell. Data Acq. and Advanced Comp. Systems: Tech. and Apps. Sofia, Bulgaria.

Fioretti, S. and Jetto, L. (1994). Low a priori statistical information model for optimal smoothing and differentiation of noisy signal. Int. J. of Adaptive Control and Signal Proc., 8, pp. 305:320.

Fossen, T. (2002). Marine Control Systems: Guidance, Navigation and Control of Ships, Rigs and Underwater Vehicles. Marine Cybernetics, Trondheim.

Gallieri, M. (2009a). Altitude Control of a Seaweed Harvester. Master's thesis, Universitá Politecnica delle Marche, Ancona, Italy. In cooperation with the National University of Ireland, Maynooth, IE.

Gallieri, M. and Ringwood, J. (2009). Optimal harvesting patterns for a seaweed harvester. In Proc. 20th Irish Sig. and Syst. Conf. Dublin, Ireland.

Gallieri, M. and Ringwood, J. (2010). Altitude control feasibility for a seaweed harvester. In Proc. of the Int. Conf. on Ind. Tech. 2010 (ICIT10), pp. 26:31. Valparaiso, Chile.

Gallieri, M. (2009b). Feasible altitude control of a seaweed harvester. Technical report, Dept. of Electronic Eng., Nat. Univ. of Ireland, Maynooth.

Hasselmann, K. and Olbers, D. (1973). Measurements of wind-wave growth and swell decay during the joint north sea wave project (JONSWAP). Ergnzung zur Deut. Hydrogr. Z., Reihe A, 8(12), pp. 1:95.

Hsiao-Ping, H. and Yung-Cheng, C. (1982). Optimal tuning of a practical digital pid controller. Chemical Eng. Commun., 18, pp. 51:61.

Jazwinski, A.H. (1970). Stochastic process and filtrering theory. Academic Press, New York.

Journee, J.M. and Massie, W.W. (2001). Offshore Hydromechanics - $1^{\text {st }}$ Edition. Delft Univ. of Technology.

Kristiansen, E. and Egeland, O. (2003). Frequency dependent added mass in models for controller design for wave motion ship damping. In 6th IFAC Conf. on Manoeuvring and Control of Marine Craft (MCMC). Girona, Spain.

Ljung, L. (1999). Identification: Theory for the user. Prentice-Hall, 2 edition.

Messineo, S., Celani, F., and Egeland, O. (2008). Crane feedback control in offshore moonpool operations. Control Eng. Practice, 16, pp. 356:364.

MSS (2010). MSS Marine Systems Simulator. Viewed 14.07.2009, http://www.marinecontrol.org.

O'Cathain, M., Leira, B., Ringwood, J., and Gilloteaux, J.C. (2008). Modelling of multibody marine systems with application to wave-energy devices. Ocean Eng., 35 , pp. 1381:1387.

Perez, T. (2005). Ship motion control. Course keeping and roll stabilization using rudder and fins. Springer.

Sagatun, S., Johansen, T., Fossen, T., and Nielsen, F. (2003). Wave synchronizing crane control during water entry in offshore moonpool operations. IEEE J. Oceanic Eng., 28, pp. 720:728.

Seatex (2006). Seatex MRU User's Manual, 11 edition.

Wamit (2008). WAMIT User Manual, Version 6.4, 6.4PC, 6.4S, 6.4S-PC. Wamit inc. Available online on www.wamit.com. 